\title{
The induced effect, vertical disparity, and stereoscopic theory
}

\author{
BARBARA GILLAM \\ State University of New York, State College of Optometry, New York, New York \\ and \\ BO LAWERGREN \\ Hunter College of the City University of New York, New York, New York
}

\begin{abstract}
The induced effect is an apparent slant of a frontal plane surface around a vertical axis, resulting from vertical magnification of the image in one eye. It is potentially important in suggesting a role for vertical disparity in stereoscopic vision, as proposed by Helmholtz. The paper first discusses previous theories of the induced effect and their implications. A theory is then developed attributing the effect to the process by which the stereoscopic response to horizontal disparity is scaled for viewing distance and eccentricity. The theory is based on a mathematical analysis of vertical disparity gradients produced by surfaces at various distances and eccentricities relative to the observer. Vertical disparity is shown to be an approximately linear function of eccentricity, with a slope or gradient which decreases with observation distance. The effect of vertical magnification on such gradients is analyzed and shown to be consistent with a change in the eccentricity factor, but not the distance factor, required to scale horizontal disparity. The induced effect is shown to be an appropriate stereoscopic response to a zero horizontal disparity surface at the eccentricity indicated. However, since extraretinal convergence signals provide conflicting evidence about eccentricity, they may attenuate the induced effect from its mathematically predicted value. The discomfort associated with the induced effect is attributed to this conflict.
\end{abstract}

In binocular vision, the consequence of vertically magnifying one retinal image (usually done with an afocal lens) is an apparent slant of a frontal plane surface around a vertical axis (Ogle, 1950). This mysterious, apparently stereoscopic phenomenon is known as the induced effect. It appears similar, but opposite in sign, to the geometric effect, a slant around a vertical axis that occurs when one image is magnified horizontally (see Figure 1). However, there is no straightforward geometric explanation in the case of the induced effect. Since the eyes are separated horizontally, not vertically, a depth difference between two points produces a horizontal, not a vertical, disparity in their images. In the real world, no arrangement of objects on the median plane would produce vertical disparity. Therefore, the induced effect cannot be predicted by triangulation of the lines of sight as the geometric effect can. Ogle (1938, $1939,1950)$, who has investigated the induced effect

Much of this paper was presented at the Annual Meeting of the American Academy of Optometry, Portland, Oregon, 1976 (Gillam \& Lawergren, 1976). B. Gillam's mailing address is: SUNY, State College of Optometry, 100 E 24th Street, New York, New York 10010. B. Lawergren's is: Hunter College of CUNY, 695 Park Avenue, New York, New York 10021.

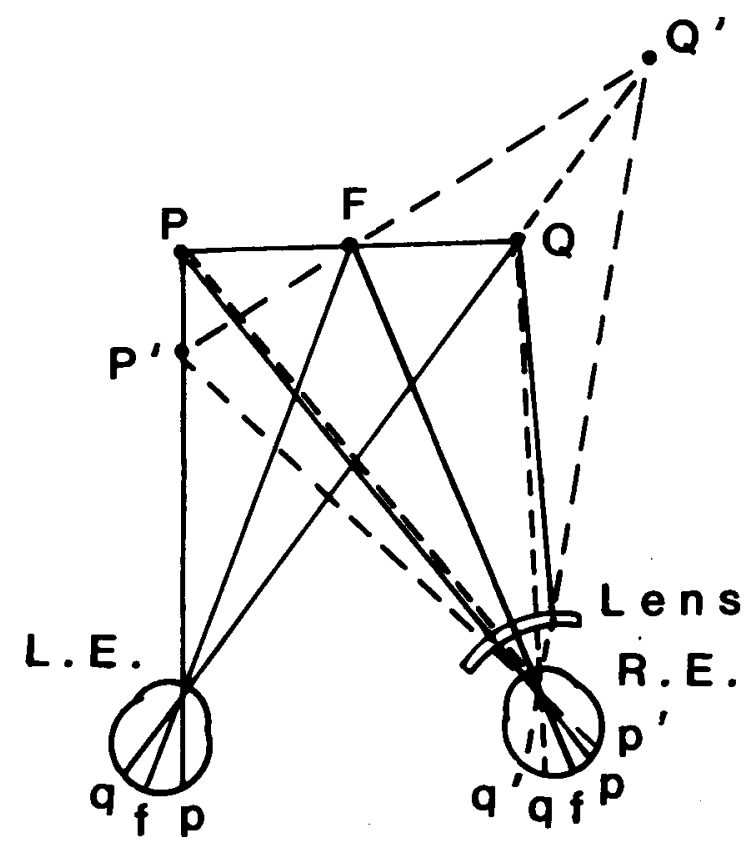

Figure 1. Geometric optics involved in the stereoscopic distortion of surfaces when a lens magnifying in the horizontal meridian is placed before one eye (from Ogle, 1950). 
most thoroughly, reports the following facts about it.

(1) It is opposite and equal to the geometric effect for small magnifications, but peaks at about $6 \%$ magnification and declines at higher magnifications (Ogle, 1938).

(2) It requires vertically separated contours in the field of view, but does not depend on their separation.

(3) The presence of as few as two vertically separated contours can cause an entire field of horizontally separated vertical horopter rods to appear slanted when vertical disparity is introduced, although the rods do not in themselves provide details that can become vertically disparate. However, the effect is greater when multiple vertically separated contours are present.

(4) As in the case of the geometric effect, the induced effect increases with observation distance (Ogle, 1938).

It should be added that the effect appears to be absent for certain stimulus configurations even when vertical disparity is present (Arditi, Kaufman, \& Movshon, 1981; Westheimer, 1978).

\section{THEORIES OF THE INDUCED EFFECT}

Since the induced effect appears to be a stereoscopic phenomenon, the explanation must be sought among those factors which affect stereopsis: (a) horizontal disparity, which can be defined in many ways but in general refers to a difference in the horizontal dimensions of the two retinal images of a given configuration, and (b) the scaling factors necessary to interpret or respond to disparate images.

Horizontal disparity in itself is ambiguous, since the same disparity can be produced by any one of a whole family of pairs of points whose depth separations increase with the square of their mean distance from the observer. Wallach and Zuckerman (1963) discussed this distance effect and introduced the concept of "stereoscopic depth constancy" to refer to it.

Horizontal disparity is also affected by the lateral position of the viewed object, a factor which may be more relevant to the induced effect. To keep disparity constant when lateral position is varied, surface slant must also be varied. Ebenholtz and Paap (1973) and Miller and Ogle (1964) have shown that the depth response to disparity does indeed change when objects are placed eccentrically.

The first theory to be discussed attributes the induced effect to the influence of vertical disparity on horizontal disparity per se, whereas the other two theories under consideration attribute it to the influence of vertical disparity on the registration of lateral position and therefore on the way horizontal disparity is scaled.
(1) The horizontal disparity theory. Westheimer (1978) suggested an explanation of the induced effect, which has been developed more fully by Arditi (1982) and Arditi et al. (1981). They proposed that the introduction of vertical magnification produces physical horizontal disparity when oblique lines are present.

Consider Figure $2 \mathrm{~A}$ in which the oblique line $1 \mathrm{ll}^{\prime}$ is magnified vertically on the right eye to be imaged as

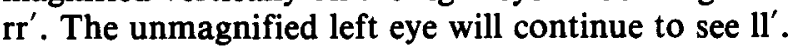
Rays from the point where the two lines cross (c) are assumed to pass through the axis of the lens (shown as a horizontal line) and to be undeviated. The vertical change associated with magnification is directly proportional to the offset of any given point from the lens axis. It can be seen in Figure 2A that the effect of vertical magnification on an oblique line is to reduce its tilt. As a result, the unmagnified eye now has the more tilted (wider) image of the line. As far as orientation disparity is concerned, vertical magnification of one image of an oblique line has the
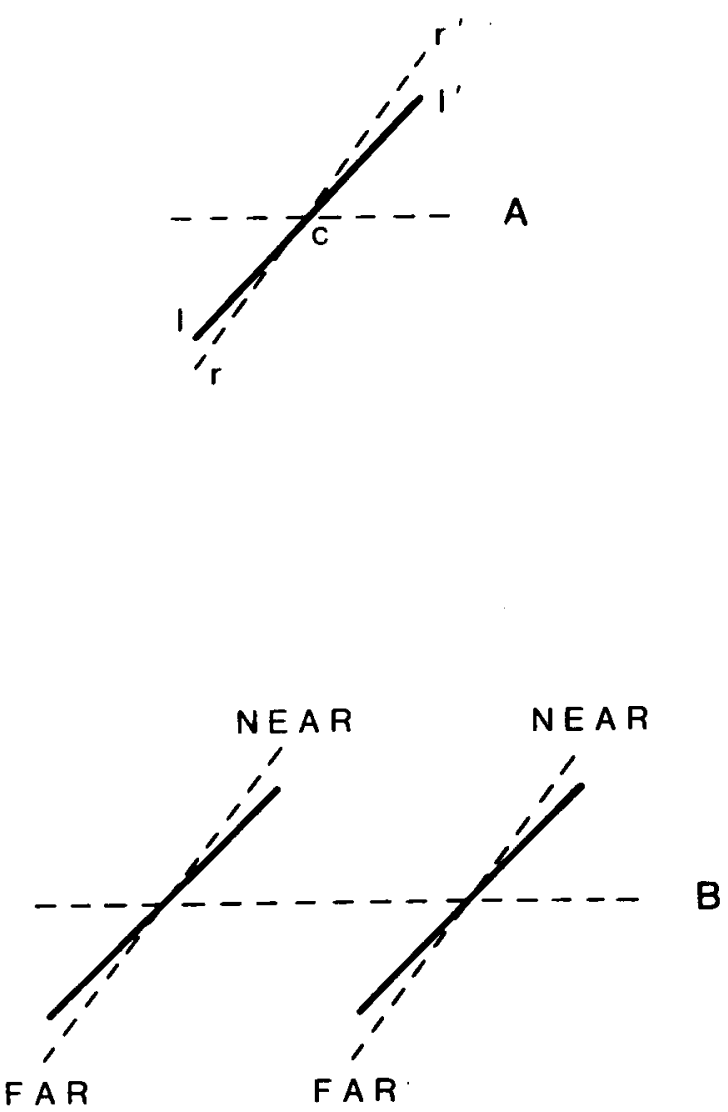

Figure 2. (A) Showing the left and right lmages (II' and rr', respectively) of an oblique line magnified vertically in the right eye. (B) The same for two oblique lines also showing the apparent depth effect resulting from the horizontal disparity present in the two images. 
same effect as horizontal magnification of the other. According to the theory, this explains why the induced effect is opposite in sign to the geometric effect. For example, in Figure $2 \mathrm{~A}$ there is a gradient of uncrossed horizontal disparity above and to the right of center and, therefore, the line will appear increasingly distant on the right.

Arditi et al. (1981) apply this analysis to a single oblique line and a pair of oblique lines crossing each other. They claim that by extending their analysis to complex patterns containing multiple lines, the induced effect, which is a slant of the entire binocular visual field, can be explained. Unfortunately, this does not work. The analysis of a single line turns out to have been misleading, since up/down is confounded with left/right in that case, and the axis of slant is ambiguous. When multiple oblique lines are examined (Figure 2B), it is obvious that vertical and horizontal disparity are both invariant along any given horizontal meridian and that both vary as a gradient along any vertical meridian. The slant of the surface will therefore be in the up-down direction (around a horizontal axis) and not in the left-right direction (around a vertical axis) as is the case for the induced effect. This was also recently pointed out by Mayhew and Frisby (1982).

Despite its failure to predict the induced effect, the theory of Arditi et al. makes a legitimate point in drawing attention to the horizontal disparities introduced by vertical magnification of oblique lines. Such disparities ought to produce local depth effects in complex patterns magnified vertically. The reasons why this does not appear to happen need to be considered. For example, if two lines of opposite orientation are viewed with vertical magnification of one eye, the theory predicts and observation confirms that their upper ends will point in opposite directions in depth. If such lines happen to cross at the center (Figure 3A), this will be consistent with the induced effect. If they have any other configuration relative to each other, such as occurs in Figure 3B, they will be stereoscopically nonplanar. Were the unbroken oblique lines in Figure 3B to be extended until they met, the apex would take on different depths, depending on which of the right-eye images the single left-eye image is related to in calculating the horizontal disparity.

Why are nonplanar resolutions of the kind predicted above not seen when vertical magnification is applied to complex planar patterns containing oblique lines? One reason may be the presence of depth ambiguities, with opposite resolutions canceling each other out. An even more important reason may be that, considered globally, the set of vertical and orientation disparities produced by this operation are consistent with a planar resolution, but one which has a different slant and eccentricity from the original stimulus. This will become clearer when the next group of theories is considered.

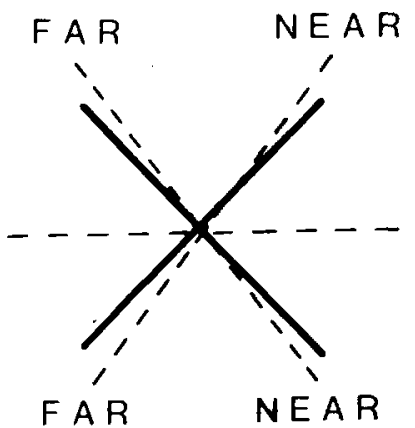

A

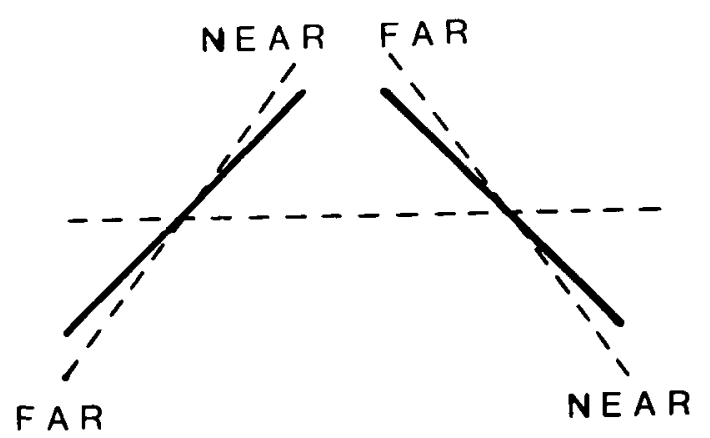

Figure 3. The left (unbroken) and right (dotted) images of oblique lines after vertical magnification of the right-eye view. The apparent depths predicted on the basis of horizontal disparity are shown.

(2) Asymmetric convergence theories. A second group of theories attributes the induced effect to (a) a misregistration of the lateral position of the viewed plane, and (b) a consequent change in the way horizontal disparity is interpreted. There are two such theories which agree on the general reason why vertical disparity causes apparent slant in an unslanted surface. Both hold that its presence causes the surface to be processed stereoscopically as if placed eccentrically in the field of view. The two theories differ, however, concerning the mechanism by which this occurs.

Vertical disparity can be considered a cue for lateral position in the field of view, since it occurs only when the object is located to the side of the median plane and therefore at a different distance from each eye. Horizontal disparity, on the other hand, depends on the orientation of the plane, as well as its lateral position, and can be present for centrally placed surfaces. Although vertical disparities are in a sense independent of eye direction (the eyes can be thought of as scanning across optic arrays with given vertical disparities), it is nevertheless true that vertical disparity of a fixated contour would occur only if the eyes were asymmetrically converged on it. Therefore vertical 
disparity at the fixation point may be especially important in signaling eccentric placement of a surface. However, the pattern of vertical disparities on a frontal plane surface is also an indication of convergence state. When convergence is symmetric, objects to the left of the fixation point will have larger left-eye images, whereas points to the right of fixation will have larger right-eye images. However, when an object is placed entirely to one side of the median plane or when the image in one eye is magnified vertically with a lens, one eye will have a vertically larger image on both sides of the fixation point.

The two theories of the induced effect which connect it to asymmetric viewing are interesting in the different assumptions they make about the nature of stereoscopic processing, since they represent two antagonistic traditions in this area: that of Hering (1879), who emphasized a sensory basis for stereopsis in the form of "local signs" for depth, and that of Helmholtz (1909), whose more empirical approach was concerned with accounting for veridical perception and specifying in a general way the information necessary to achieve it. These theories will be called the "normal horopter theory" and the "stereoscopic constancy scaling theory," respectively (terms coined by the present authors).

\section{The Normal Horopter Theory}

This was developed by K. N. Ogle and is based upon Hering's (1879) assertion that points which stimulate corresponding points in the two eyes appear to be at equal depth. By "equal depth," Hering meant the frontal plane or the tangent to the ViethMuiller circle at the fixation point. The appearance of equal depth is assumed to be a sensory response to the stimulation of corresponding points and, as such, should apply under all viewing conditions.

However, the idea runs into problems in asymmetric convergence when the fixation point is not on the median plane. As Ogle (1950) points out, and as shown in Figure 4, under these conditions lines tangent to the Vieth-Muiller circle are not normal to the line of sight (the general case of perceived equidistance), which means that there is no longer a geometric relationship between correspondence and equidistance. To restore this relationship under conditions of asymmetric convergence would require that points physically on the tangent ( $\left.\mathrm{tt}^{\prime}\right)$ be perceptually rotated so that they appear on the normal (nn') (see Figure 4). This idea has been proposed at least once (Miller \& Ogle, 1964). However, it would lead to gross perceptual error and, in any case, is of no interest here because the rotation would be towards the vertically magnified eye-the opposite of the induced effect.

Hering's theory could be saved and perceptual error avoided if points physically on the normal to the line of sight were made to fall on corresponding points

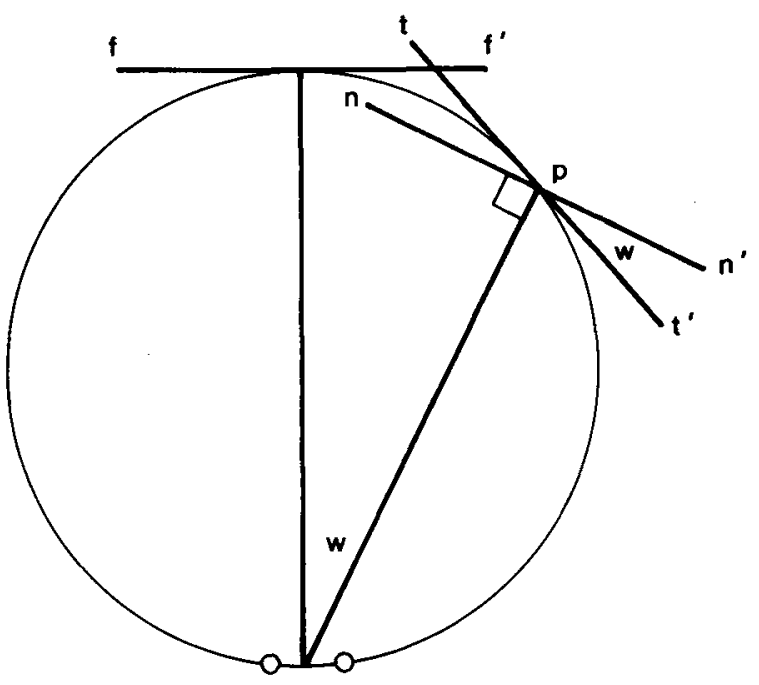

Figure 4. Showing the frontal plane, ff', the normal plane, $\mathrm{nn}^{\prime}$, and the tangent to the Vieth-Müller circle (tt') for eccentric viewing (by $w$ degrees) on $P$. This angle will henceforth be referred to as the angle of eccentricity. Note that the deviation of the normal from the tangent to the Vieth-Müller circle (Ogle's prediction for the induced effect) is also $\mathbf{w}$ degrees.

by magnifying the more distant eye's image in the horizontal meridian (the left image in Figure 4). Points on the normal would now appear normal by virtue of stimulating corresponding points. The magnification process could be triggered by some indication that the configuration was laterally displaced. This could be information provided either by extraretinal convergence signals or by the presence of vertical disparity for contours at the fixation point. Ogle assumes that the latter is sufficient and that the necessary horizontal magnification is automatically achieved by overall magnification of the eye with a smaller vertical image, of the degree required to eliminate the vertical difference. An automatic overall magnification of the image with the smaller vertical extent, if applied in symmetric convergence or central viewing where no correction is needed to place the surface on corresponding points, would result in the induced effect. Its predicted magnitude would be equal but opposite to the geometric effect resulting from horizontal magnification of the same degree. In a sense, this version of Ogle's theory could be regarded as a horizontal disparity theory, since it proposes that disparity itself, and not its interpretation, is altered by the simulation of asymmetric convergence.

Ogle (1950) reports, however, that in measurements of corresponding points by the nonius method under conditions of asymmetric convergence, no such compensatory magnification is evident. Miller and Ogle (1964) also failed to show any evidence of compensatory magnification in a study in which afterimages of lights in the frontal plane were ob- 
tained in symmetric convergence and their apparent slant measured in asymmetric convergence. Adding vertically separated contours to the target controlling convergence did not help. Ogle concludes, as a result of these and other experiments, that the compensatory effect operating in asymmetric convergence must be a psychological rotation of the equal depth reference surface from the Vieth-Müller tangent ( $\mathrm{tt}^{\prime}$ ) to the normal (nn') (see Figure 4). He says it is as if the image had been magnified horizontally in the more distant eye, that is, the normal is now responded to perceptually as if it were stimulating corresponding points.

Although Ogle does not present it this way, this position is really an abandonment of Hering's theory, since it implies that in asymmetric convergence the perception of equal depth is not tied to the stimulation of corresponding points. Morrison (1977) discusses some of the issues involved here and explicitly rejects the idea of a rigid connection between stimulation of corresponding points and the sensation of a normal plane, since subjects are able to set rods correctly to the normal in asymmetric convergence even though the setting requires the rods to be disparate.

One might go a step further and propose that even in symmetric convergence perception of the normal or the frontal plane is not a sensation elicited by the stimulation of corresponding points (Ogle, 1950; Hering, 1979), but a percept which is mediated by different image relationships (disparities) at different distances and which is likely to involve learning. This was Helmholtz's position and will be further elaborated on in connection with the next theory to be discussed.

\section{Stereoscopic Constancy Theory}

This class of theory assumes no a priori relationship between correspondence and perceived equidistance. It is assumed that the horizontal dimensions of the images on the two retinas, whether the same or different, are interpretable as objects in depth only through mathematical relationships involving the distance and lateral position under which the images arise. ${ }^{2}$ Theories that have used this nonphysiological approach to explain the induced effect include those of Gillam and Lawergren (1976), Householder (1943), Petrov (1980), and, most recently, Longuet-Higgens and Mayhew (1982). We present our own version below. The important question for this approach concerns the information used to obtain the necessary estimates of distance and lateral position.

Because it is always associated with lateral displacement of an object, vertical disparity is assumed to be sufficient to influence registration of the lateral position factor and, if introduced artificially, to cause it to be misregistered or biased and depth relationships to be misperceived. Vertical disparity does not, however, cause actual misperception of direction of gaze. [This is directly analogous to the theory that geometrical illusions arise because of the inappropriate application of three-dimensional perspective processing to two-dimensional displays, given the presence of certain features normally associated with perspective projection, even though the displays do not appear three-dimensional (Gillam, 1980; Gregory, 1963)].

Figure 5 illustrates the assumptions of the stereoscopic constancy theory in a more concrete way. Horizontal disparity alone cannot distinguish between $\mathrm{ff}^{\prime}$, a frontal plane, and $\mathrm{ss}^{\prime}$, a slanted plane, which both subtend equal horizontal angles in the two eyes. However, the center (c) of $\mathrm{ff}^{\prime}$ is equidistant from the two eyes and would have zero vertical disparity (assuming it to have vertical extent), whereas the center (d) of ss' is not equidistant and will have a finite vertical disparity, with the image sizes on the eyes proportional to their distances from $d$. Therefore, the degree of vertical disparity could be the key factor in determining whether the response to equal horizontal angles will be a frontal plane (which is appropriate for central viewing) or a plane slanted towards the eye with the larger vertical image (which is appropriate for eccentric viewing). Such a process would account for the induced effect, should vertical disparity be misleadingly introduced in central viewing. Petrov (1980) similarly attributed the induced effect to the use of "optical signals" as opposed to signals for eye position to "synthesize the visual field."

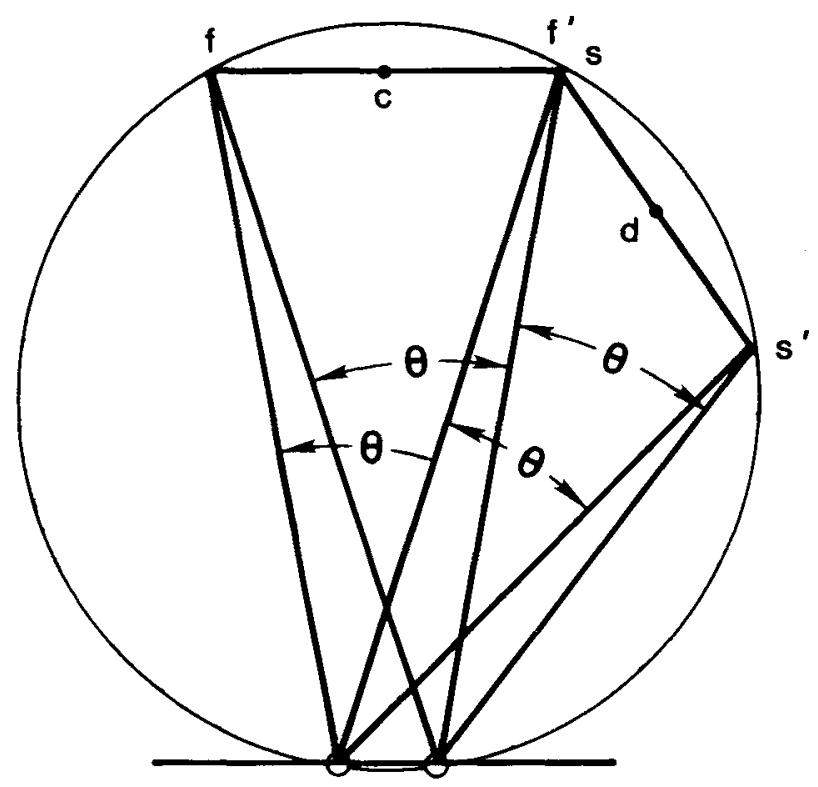

Figure 5. Illustrating the fact that in eccentric viewing equal horizontal angles are subtended at the two eyes by a slanted plane (ss'), whereas in central viewing the same equal horizontal angles are subtended by a frontal plane (ff'). 
Figure 6 shows a series of circles, each representing the loci in space over which a given ratio of distances to the two eyes and therefore a given vertical magnification of the right image relative to the left is maintained. ${ }^{3}$ A given vertical magnification can arise from any position on the appropriate circle. The derivation of the circles is given in the Appendix. In Figure 6, the radiating lines each represent loci at which vertical surfaces of a given slant around a vertical axis would produce equal horizontal angles in the two eyes. The appropriate slant (relative to the frontal plane) is shown on each line. It is always approximately twice the angular deviation of the line from the median plane ( $w$ in Figure 4). (See Appendix for derivation). The intersections of the circles with the radiating lines show approximately what slant relative to the frontal plane will give zero horizontal disparity for a given magnitude of vertical disparity

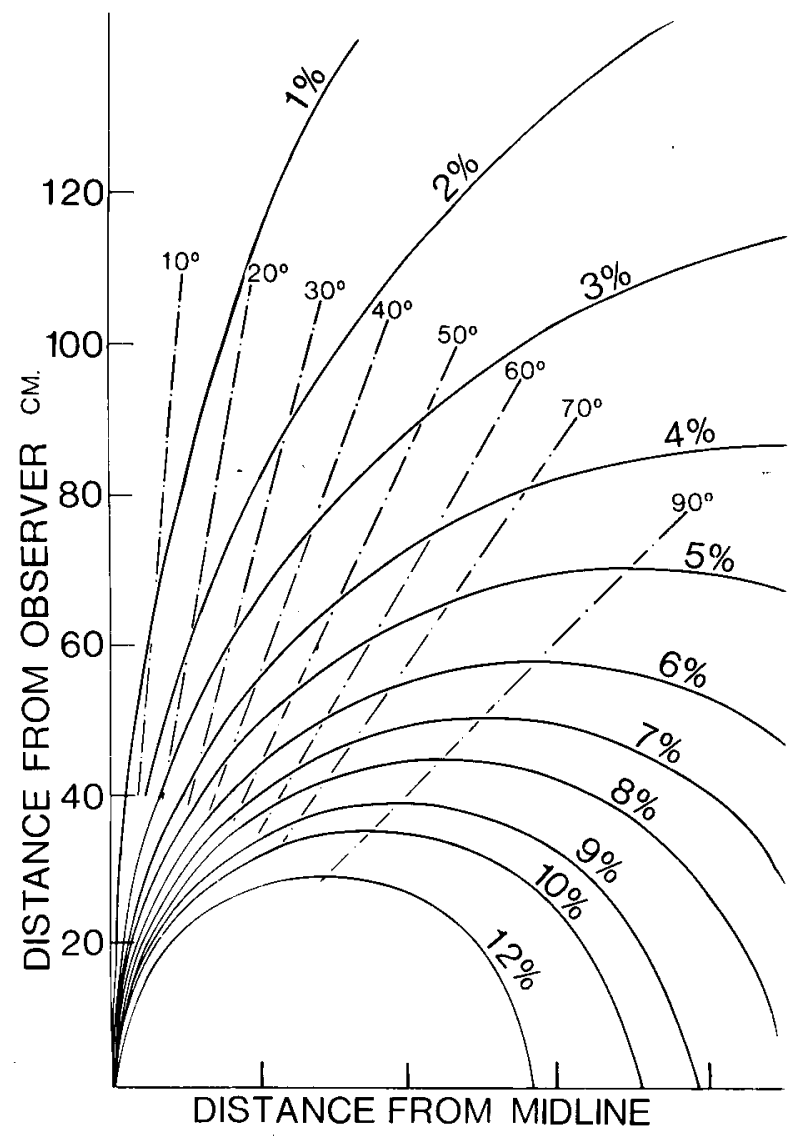

Figure 6. Showing the loci (circles) which would result in various percentage magnifications of the right-eye image relative to the left (from 1\% to 12\%). The radiating lines represent loci at which horizontal lines slanted away from the frontal plane by the number of degrees shown on the line would give rise to equal horizontal images in the two eyes. The $x$ axis represents distances along the frontal plane, and the $y$ axis represents distances along the median plane, with a point midway between the eyes as origin. Both axes have the same scale. at a given observation distance. This slant will represent the optimum induced effect for that vertical disparity at that distance. From Figure 6, it is clear that observation distance must be taken into account, since vertical disparity alone does not specify a unique spatial position and therefore does not specify a unique slant for a zero horizontal disparity surface associated with it.

How can the absolute distance factor be obtained and how would vertical magnification of one eye's image affect it? Apart from registered binocular parallax, which has been investigated by Ono and Comerford (1977), Wallach, Frey, and Bode (1972), Wallach and Zuckerman (1963), and most thoroughly by Foley (1980), there is information in the visual images themselves specifying absolute distance. This has historically been overlooked, despite the fact that Helmholtz emphasized it a great deal (but see LonguetHiggins \& Mayhew, 1982). Helmholtz argued that the curved settings obtained when subjects are required to set horopter rods to the frontal plane, which are usually considered sensory in origin (Hering, 1879; Ogle, 1950), are actually artifacts which arise from the absence of vertical disparity information in the horopter apparatus. He claims that these errors disappear when vertical disparity is made available and absolute distance is perceived veridically. ${ }^{4}$

Helmholtz does not spell out the mathematical nature of the absolute distance information available from vertical disparity. However, it is quite simple. Figure 7 shows the ratio of vertical images in the two eyes as a function of angle of eccentricity across a series of frontal planes. The planes are assumed to be bisected by the visual plane and to originate at the median plane, where the ratio is 1 . Observation distance is the parameter, with distance increasing from the top to the bottom of the figure. There is a nearly linear increase in vertical disparity (defined here as the ratio of tangents for the vertical visual angles rather than their difference) as a function of angular eccentricity. The slope of the function decreases with absolute distance. Since slope is independent of eccentricity, it could be used to evaluate absolute distance.

Figure 8 shows what happens to one of the functions of Figure 7 when the entire retinal image is magnified vertically by a constant percentage. It can be seen that such a magnification does not change the slope of the function, ${ }^{3}$ and therefore does not change the information about absolute distance. It changes only the intercept, which makes the new function equivalent to the old one at a greater eccentricity (see Figure 8).

In one important respect, vertical disparity behaves quite unlike horizontal disparity. Local depth changes, including quite large slants away from a frontal plane position, do not change vertical dispar- 


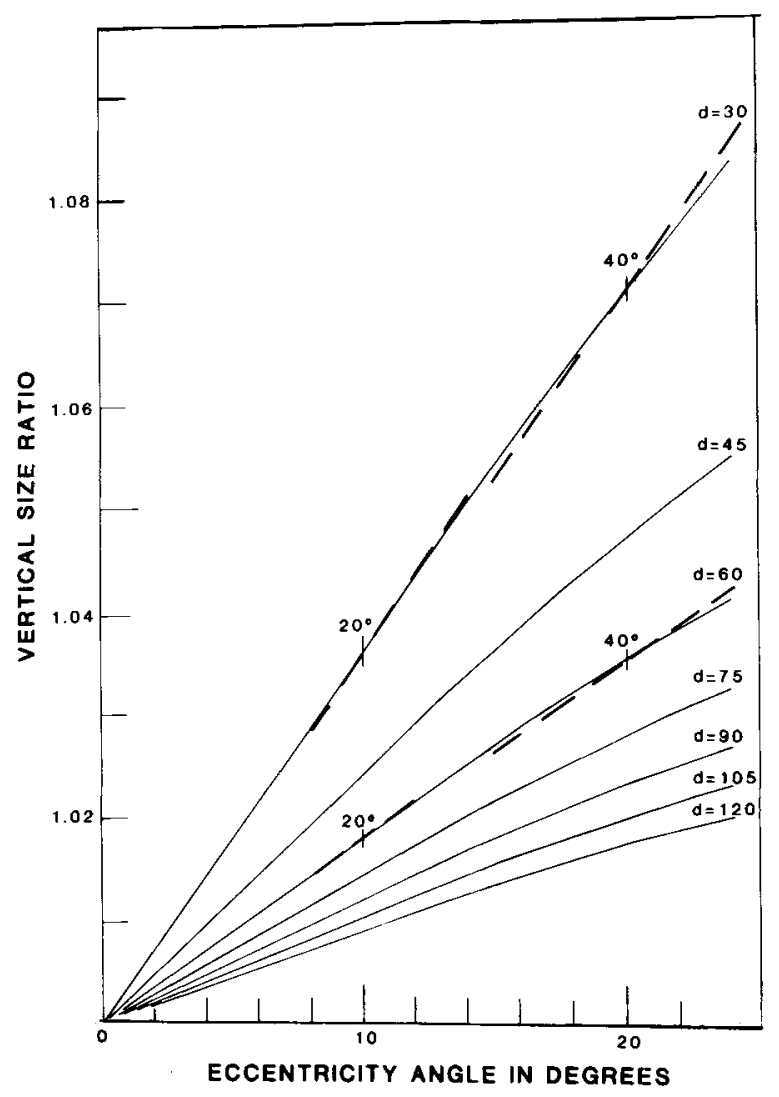

Figure 7. The ratio of vertical image sizes in the two eyes of vertical lines on a frontal plane centered on the visual plane at various eccentricities relative to the median plane. The parameter is the distance of the frontal plane (measured along the median plane). The dashed lines each represent the vertical size ratios when the plane is slanted around an axis at the position of eccentricity and distance indicated by the short vertical line. The degree of slant relative to the frontal plane is shown.

ity functions (either slope or intercept) to any significant degree. The dashed lines in Figure 7 represent the ratios of the tangents for vertical visual angles along planes which are slanted away from the frontal plane around various eccentric axes, as indicated, so as to be normal to the direction of the axis from the interocular midpoint. To indicate the trend, only four axis positions are shown, namely at distances of 30 and $45 \mathrm{~cm}$ and eccentricities of 10 and $20 \mathrm{deg}$. Slants relative to the frontal plane are shown at each axis position. It is obvious that the ratio function (see Appendix) changes very little when the plane shifts from a frontal to a markedly slanted position. This shows that vertical disparity provides information about the absolute distance and eccentricity of a surface independently of its orientation.

As far as the induced effect is concerned, the main points following from the above analysis are:

(1) The slope of the gradient of vertical disparity could potentially provide the absolute distance infor- mation needed to locate a unique eccentric spatial position for the source of an individual vertical disparity.

(2) Introducing a vertical magnification by means of lenses does not alter this absolute distance information. It effectively produces the vertical image size ratios of a more eccentrically placed surface at the same distance.

(3) If registered eccentricity is influenced by vertical image size ratios, this is sufficient to explain the perception of zero horizontal disparities as slanted (induced effect).

(4) The vertical disparity gradient does not provide any significant information about local depth or slant and therefore does not in itself support seeing a surface at its true slant.

(5) Although some slant of an array of horopter rods is induced by a single pair of vertically disparate contours on the fixation rod (Amigo, 1972; Ogle, 1950), the enhancement of the induced effect in the presence of multiple vertically separated contours (Ogle, 1950) is not surprising, since in the latter case the entire gradient supports an eccentric placement of the surface.

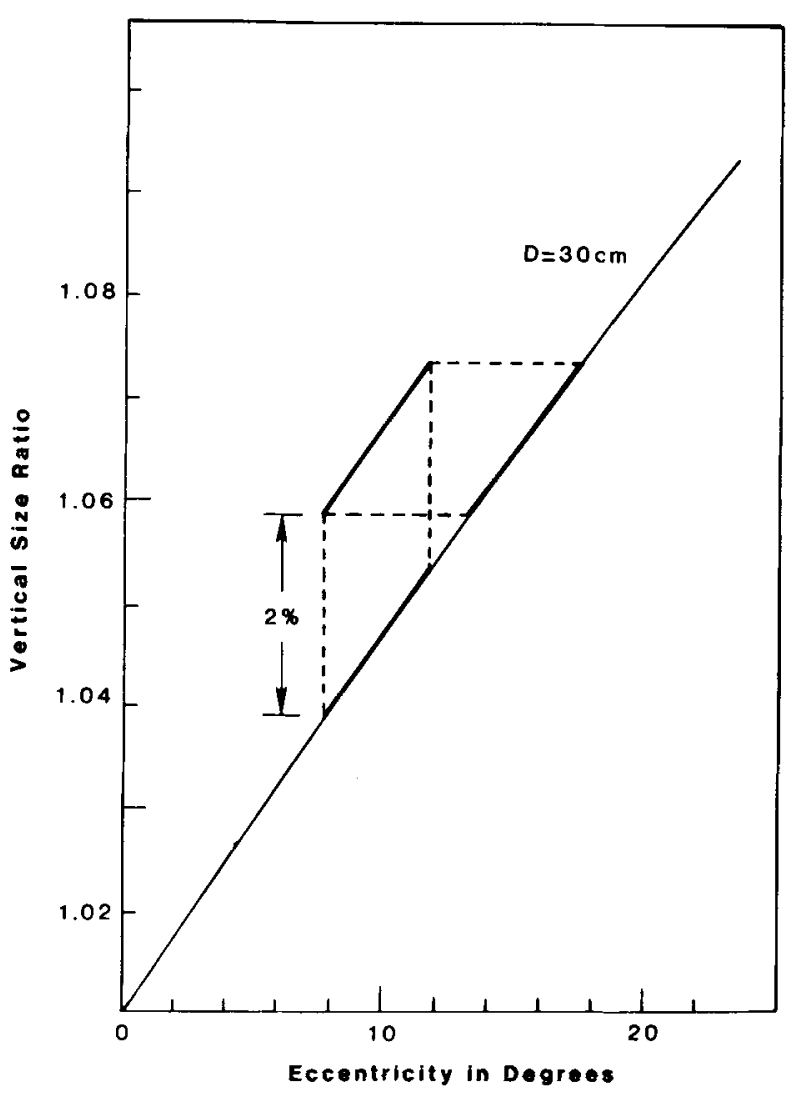

Figure 8. Showing the effect on vertical size ratios of magnifying the right-eye image by $2 \%$ for the $30-\mathrm{cm}$ function. Note that only the intercept changes, making this function equivalent to one at the same distance but greater eccentricity. 
It is obvious from both Figure 6 and Figure 7 that large vertical disparities simply do not arise at greater distances. For each distance, there is a disparity limit. Therefore, assuming that the system has developed to handle the vertical disparities that would arise in normal viewing, the induced effect would be expected to be restricted to relatively small vertical disparities. This should be increasingly the case at greater observation distances, where large vertical disparities never occur naturally. The induced effect does have an asymptotic value at rather low magnifications (Ogle, 1950). This will be discussed further in the section on measurements of the induced effect.

So far, we have assumed that the slant induced by a given vertical magnification would be that which would produce images with zero horizontal disparity at the spatial position specified by the vertical disparity present. As can be seen from Figure 6 and as derived in the Appendix, this is always approximately twice the angle of eccentricity (w), or twice the effect predicted by Ogle. [Ogle assumes that the rotation induced is of the degree necessary to alter the stereoscopic reference surface in asymmetric convergence from the tangent to the Vieth-Muiller circle at $p$ to the normal at $p$ (Figure 4), whereas we propose that it is of the degree necessary to shift the perceived slant of a zero horizontal disparity surface from the frontal plane, $\mathrm{ff}^{\prime}$, to the Vieth-Müller tangent, $\mathrm{tt}^{\prime}$ (Figure 4).] However, vertical disparity alone would only be expected to operate fully in stereopsis as an indication of the surface eccentricity and the convergence state of the eyes if extraretinal signals played no significant role in registering eccentricity or convergence states. This does not seem likely, since it has been shown many times (Amigo, 1972; Ebenholtz \& Paap, 1973; Miller \& Ogle, 1964) that asymmetric convergence without vertical disparity can have a considerable influence on the interpretation of horizontal disparity. It is also interesting in this regard that vertical disparity introduced by means of a lens is uncomfortable, producing complaints of eye strain, dizziness, or even nausea (Ogle, 1938). This strongly indicates a conflict between the asymmetric convergence state indicated by the presence of vertical disparity at the fixation point and the symmetric convergence state actually required for fixation.

Charnwood (1950) suggests that clinicians prescribe prism for the asthenopic symptoms associated with aniseikonia. This presumably gives relief because it brings the two conflicting convergence indicators, namely, muscle "outflow" and vertical disparity, into harmony. The vertical disparity associated with real asymmetric convergence causes no discomfort, since it is compatible with any convergence information provided by muscular states.

In the light of the evidence previously mentioned, that extraretinal convergence signals have some effect on stereoscopic processing, the induced effect might be expected to be considerably less than predicted mathematically for the position specified by the vertical disparity in force. It would also be reasonable to expect that the greater the eccentricity angle indicated by the vertical disparity and therefore the greater the conflict between it and extraretinal convergence information, the more the observed induced effect would be expected to deviate from the one predicted from vertical disparity alone.

\section{INTERPRETATION OF MEASUREMENTS OF THE INDUCED EFFECT}

On the whole, the stereoscopic constancy theory is consistent with the known properties of the induced effect. The effect increases with magnification and observation distance as predicted and peaks at low magnifications relative to the geometric effect (Ogle, 1950). As stated earlier, the magnification at which the maximum occurs might be expected to decrease with observation distance. Few data are available on this point. Ogle (1938) varied distance for only one subject and found maximum induced effects at magnification values of $10 \%$ and $12 \%$ (for right- and left-eye magnifications, respectively) for the $20-\mathrm{cm}$ distance and at $8 \%$ and $6 \%$ magnifications for the $75-\mathrm{cm}$ distance. This does provide some support for the hypothesis of a decline in optimum magnification for the induced effect as distance increases. However, it is clear that more research is needed on this important point. We are currently conducting a series of experiments (to be reported in a subsequent publication) in which distance is among the parameters varied.

It is not surprising that vertical disparity of elements on the fixation rod alone is found to be sufficient to slant the whole visual field if the rest of the field does not contain vertically separated contours (Amigo, 1972; Ogle, 1950). A vertical disparity for the only vertically separated contours present would indicate a state of eccentricity for the stimulus. On the other hand, the introduction of vertical disparity for some, but not all, vertically separated elements would not be consistent with eccentric viewing and might therefore not lead to an induced effect. This may be the reason for the lack of an effect reported by Arditi et al. (1981) for vertically disparate vertical lines enclosed in nondisparate boxes. Westheimer (1978) claims that his stimuli are simple and therefore likely to elicit a more sensory response than Ogle's. He found no induced effect. This might have been due to the brief exposure durations he used, to the presence of nondisparate vertical elements in the field, or to the fact that his targets, though simple, formed rectilinear configurations which provide perspective information that the surface is in the frontal plane (Gillam, 1968; Ogle, 1938). Ogle's random-dot targets avoid this complication. 
Ogle found that the induced effect was as great in magnitude as the geometric effect up to about $6 \%$ magnification. Since this is his prediction for a maximum effect, this means that, according to his theory, vertical disparity is sufficient, despite conflicting extraretinal convergence information, to trigger the full effects of asymmetric convergence. On the other hand, Ogle's data represent a slant considerably less than that required to produce zero horizontal disparity at an eccentricity consistent with the vertical magnification given, suggesting that conflicting convergence signals may be attenuating the effect of vertical disparity in signaling eccentricity.

\section{CONCLUSION}

Given that eccentricity in the visual field must be registered in order to process horizontal disparity, and given that vertical disparity, especially vertical disparity gradients, provide robust information about eccentricity, and given that a misregistration of eccentricity in accordance with the vertical disparity given would explain the perceived slant known as the induced effect, it is plausible to account for the induced effect as an illusion of misregistered eccentricity. It is especially notable that the vertical magnification of one retinal image by a lens exactly mimics the effect on gradients of vertical disparity of changing their lateral position in the field. This explanation of the induced effect is consistent with existing data and avoids postulating special mechanisms to account for the effect. If the information potentially provided by vertical disparity is used in spatial localization, then the induced effect must follow, unless vertical disparity information is entirely overcome when placed in conflict with extraretinal convergence signals, instead of reinforcing them, as it would do in normal vision. It is worth noting that many of the "disparity detectors" in the visual cortex respond to vertical disparity (Bishop, 1975), suggesting that it has some use.

\section{REFERENCES}

Amigo, G. The stereoscopic frame of reference in asymmetric convergence of the eyes. Response to "point" stimulation of the retina. Optica Acta, 1972, 19, 993-1006.

ARDit, A. The dependence of the induced effect on orientation and a hypothesis concerning disparity computation in general. Vision Research, 1982, 22, 247-256.

Arditi, A., Kaufman, L., \& Movshon, J. A. A simple explanation of the induced size effect. Vision Research, 1981, 21, 755-764.

Bishop, P. Binocular vision. In C. R. Moses (Ed.), Adler's physiology of the eye (6th ed.). St. Louis: Mosby, 1975.

Charnwood, Lord. An essay in binocular vision. New York: Hafner, 1950, 1965.

Eannholtz, S. M., \& PaAP, K. N. The constancy of object orientation: Compensation for ocular rotation. Perception \& Psychophysics, 1973, 14, 458-470.
Foley, J. M. Binocular distance perception. Psychological Review, 1980, 87, 411-434.

Gillam, B. Perception when stereopsis and perspective conflict: Experiments with aniseikonic lenses. Journal of Experimental Psychology, 1968, 78, 299-305.

Gillam, B. Geometrical illusions. Scientific American, 1980, 242(1), 102-111.

Gillam, B., \& Lawergren, B. The induced effect revisited. American Journal of Optometry and Physiological Optics, 1976, 53, 531. (Abstract)

GrEGoRY, R. Distortion of space as inappropriate constancy scaling. Nature, London, 1963, 119, 678.

Helmholtz, H. Handbuch der physiologischen Optik (3rd ed., Vol. 3; J.P.C. Southall, trans.). New York: Dover, 1962. (Originally published, 1909.)

Hering, E. Der Raumsinn und die Bewegungen des Auges (Carl A. Radde, trans.). Baltimore: American Academy of Optometry, 1942.

Householder, A. S. A theory of the induced size effect. Bulletin of Mathematical Biophysics, 1943, 5, 155-160.

Longuet-Higgins, H. C., \& MAYHew, J.E.W. A computational model of binocular depth perception. Nature, 1982, 297, 376-378.

Mayhew, J.E.W., \& Frisby, J. P. The induced effect: Arguments against the theory of Arditi, Kaufman, \& Movshon. Vision Research, 1982, 22, 1225-1228.

Miller, J. T., \& OGLE, K. N. Stereoscopic localization of afterimages with eyes in asymmetric convergence. Investigative Ophthalmology, 1964, 3, 339-353.

Morrison, L. C. Stereoscopic localization with the eyes asymmetrically converged. American Journal of Optometry and Physiological Optics, 1977, 54, 556-566.

OGLE, K. N. Induced size effect I: A new phenomenon in binocular vision associated with the relative sizes of the images in the two eyes. Archives of Ophthalmology, 1938, $20,604$.

OGLE, K. N. Induced size effect II: An experimental study of the phenomenon with restricted fusion contours. Archives of Ophthalmology, 1939, 21, 604.

OGLE, K. N. Researches in binocular vision. New York: Hafner, 1950.

Ono, H., \& Comerfond, J. Stereoscopic depth constancy. In W. Epstein (Ed.), Stability and constancy in visual perception. New York: Wiley, 1977.

Petrov, A. P. A geometrical explanation of the induced effect. Vision Research, 1970, 20, 407-413.

Shipley, T., \& RAwlings, S. C. The nonius horopter-I. History theory. Vision Research, 1970, 10, 1225-1262.

Shipley, T., \& Rawlings, S. C. The nonius horopter-II. An experimental report. Vision Research, 1970, 10, 1263-1299.

Wallach, H., Frey, K. J., \& Bode, K. A. The nature of adaptation in distance perception based on oculomotor cues. Perception \& Psychophysics, 1972, 11, 110-116.

Wallach, H., \& Zuckerman, C. The constancy of stereoscopic depth. The American Journal of Psychology, 1963, 76, 404-412.

WEsthe IMER, G. Vertical disparity detection: Is there an induced size effect? Investigative Ophthalmology, 1978, 17, 545-551.

\section{NOTES}

1. Strictly speaking, corresponding points are defined by the nonius (visual direction) horopter, which is shallower than the Vieth-Müller circle but more curved than the tangent (Ogle, 1950; Shipley \& Rawlings, 1970). In considering the effects of asymmetric convergence, this distinction is not important. It is probably better to consider the stimulus purely geometrically, since the relationship of depth perception to correspondence as defined by the visual direction criterion is not established.

2. The elevation of a configuration in the visual field also determines degree of binocular disparity for a given depth interval 
between two points (Ebenholtz \& Paap, 1973). To simplify matters, only surfaces centered on the visual plane are considered here.

3. In a somewhat sketchy paper, which mathematically anticipates some of the work described here, Householder (1943) derives mirror images of the circles shown in Figure 6-mirror images in the sense that for magnification of the right eye Householder's circles are to the left of the median plane whereas ours are to the right. Points on these circles, according to Householder's theory, locate the apparent median plane for various vertical magnifications of one eye relative to the other. (To locate a unique median plane, distance must be given, and Householder implicitly assumes that it is.) Ogle's subjects, according to Householder, were "locating the subjective median plane." He does not spell out the logic by which this should give rise to the induced effect. Presumably, the normal to the subjective median is perceived as the frontal plane and the real frontal plane is therefore seen as slanted in the opposite direction; hence, the induced effect. However, if we have interpreted him correctly, Householder's idea is needlessly complex. It is not necessary to first locate the subjective median in order to register eccentricity for the true median, which is the essential step in both his theory (at least implicitly) and the present one (explicitly). However, Householder does deserve credit for pioneering a mathematical analysis of the problem of vertical disparity and removing it from the tangled web of corresponding point theory.

4. Absence of vertical disparity forces the observer (who has to set a series of vertical rods to appear in the frontal plane and who must use some kind of absolute distance information, to do the task correctly) to rely on convergence for this purpose. Helmholtz claims that convergence distance is systematically underor overestimated in ways which account for the obtained deviations from the frontal plane. The horopter rods are set at disparities which would be equivalent to a frontal plane at the registered distance but which are equivalent to a curve at the actual distance. Helmholtz goes on to demonstrate the role of vertical disparity in determining the response to a given horizontal disparity by means of stereograms which all have zero horizontal disparity but whose apparent depth properties (convex or concave curvatures) depend on the vertical dimensions within the stimulus.

5 . Since the vertical size ratios due to eccentricity $(R)$ are near unity, one may write $R=1+\varepsilon$, with $\varepsilon$ near zero. For aniseikonic lenses with moderate magnification, $M=1+\delta$, where $\delta$ is near zero. Combining both effects, one obtains an effective vertical size ratio $\mathbf{R}^{\prime}=\mathbf{R} \cdot \mathbf{M} \approx 1+\varepsilon+\delta=\mathbf{R}+\delta$. Under these conditions, the curves in Figure 7 are simply pushed up (increased) an amount (M-1) (without slope change). Figure 8 shows how the identical increase without slope change also occurs at a greater eccentricity (without lens) for the same distance. The eccentricity increase is $(y \cdot \delta / 2 a)$ since the slopes of the lines in Figure 7 are $(2 a / y)$, as shown in the appendix.

\section{APPENDIX}

\section{Loci for Points Having a Constant Vertical \\ Magnification of One Eye Relative to the Other}

In order to map the visual field, a coordinate system is located with its $(x, y)$-plane in the visual plane. The eyes lie on the $\mathbf{x}$-axis at distances $\mathbf{A}$ on either side of the $y$-axis. The median plane constitutes the $(y, z)$-plane. Consider a point in the visual plane at location $(x, y)$. The distances between this point and the left and right eyes are $d_{l}$ and $d_{r}$, respectively. Then the ratio $d_{l} / d_{r}=R$ is identical to the ratio $\left[\tan \left(v_{1}\right) / \tan \left(v_{r}\right)\right]$, where $v_{l}$ is the visual angle in the left eye of a vertical object centered at $(x, y)$. Generally, these angles are small and the angles themselves can be used instead of their tangents. The locus for points with a constant $\mathbf{R}$ is

$$
(x-A k)^{2}+y^{2}=A^{2} \cdot\left(k^{2}-1\right),
$$

where $k=\left(R^{2}+1\right) /\left(R^{2}-1\right)$. If the "percentage magnification" of a lens is called $M, k=1 / M$ for small magnifications. Equation 1 represents a circle centered at $x=A k$ with radius $\mathbf{A}\left(\mathbf{k}^{2}-1\right)^{1 / 2}$. Such circles have been drawn for a number of $R$ values in Figure 6 .

\section{Planes Slanted to Give Equal Horizontal Visual Angles in the Two Eyes}

Consider a rectangular plane surface perpendicular to the visual plane. An axis, also perpendicular to the visual plane, is located at the midpoint of this surface. We shall restrict our attention to the projection of the surface onto the visual plane, that is, a straight line ("the object") in the $(x, y)$-plane rotatable around its midpoint. Define the slant of this object (u) as the angle between it and the line $y=y_{o}$ passing through the midpoint of the object. The midpoint of the line is at $\left(x_{0}, y_{0}\right)$ and its length is $2 p$. Let the object turn until it subtends the same visual angle in both eyes. Then the angle $\left(u_{0}\right)$ will be a function of $p$, the interocular distance (2A), and the midpoint coordinates:

$$
\tan u_{o}=\frac{2 x_{0} \cdot y_{o}}{A^{2}+y_{0}^{2}-x_{0}^{2}-p^{2}} .
$$

If $w_{0}$ is the angle between the $y$-axis and the radius vector to the object midpoint, that is, $\tan w_{0}=x_{0} / y_{0}$, it follows that $u_{o}$ is approximately twice the value of $w$ for typical values of $A, p$, and the coordinates.

If Equation 2 is treated as a function in the (x,y)-plane with $\tan \mathrm{u}(=1 / \mathrm{q})$ as a parameter, the loci are obtained for midpoints of objects that have been slanted to the angle $u$ in order to appear with equal visual angle in each eye:

$$
x^{2}-y^{2}+2 q x y=A^{2}-p^{2} .
$$

Consider this equation in the first quadrant of the $(x, y)-$ plane only. Generally, it represents a hyperbola, its asymptote passing through the origin forming an angle of $u / 2$ with the $y$-axis. However, if $\mathbf{p}=\mathbf{A}$, Equation 3 is simply a line identical to this asymptote. If $p>A$, the hyperbola lies above the asymptote. In the typical case, in which the object lies at least $30 \mathrm{~cm}$ away from the eyes, the hyperbola is very close to its asymptote and it is justified to draw these loci as straight lines, as in Figure 6.

\section{Magnification as a Function of Angle of Eccentricity With Distance as a Parameter}

Using Equation 1, it is easy to find a relationship between the magnification $R$ and the angle $w$

$$
R^{2}=\frac{y^{2}+(A+y \cdot \tan w)^{2}}{y^{2}+(A-y \cdot \tan w)^{2}} .
$$

For small angles and $A \ll y$, this can be simplified to $R=[(2 A / y) w+1]$, that is, $R$ and $w$ are linearly related. Figure 7 is based on Equation 4. The slopes are indeed ( $2 \mathrm{~A} / \mathrm{y})$, that is, they are characteristic for each distance $y$.

(Manuscript received November 5, 1982; revision accepted for publication April 5, 1983.) 